\title{
PEN vS. COMPUTER-How to Write by Hand Improves Memory and Creativity; Neuroscience, Rehabilitation, Psychomotor Skills
}

\begin{abstract}
Dario Furnari, ${ }^{1,2 *}$ Nadya Khan, ${ }^{3}$ Melissa Delaney, ${ }^{1}$ Margaret Cerna, ${ }^{1}$ Khaled Hamlaoui, ${ }^{1}$ Sebastien Lagree, ${ }^{1,2}$ Amy Peace, ${ }^{1,2}$ Megan Owens,,${ }^{1,2}$ Susana Sanchez, ${ }^{1}$ Aliaksandra Eyring, ${ }^{1}$ Elena Talyanova, ${ }^{1}$ Monika Milczarek, ${ }^{4}$ Abdulrahim Aljayar, ${ }^{1}$ James Stoxen, ${ }^{1}$ Sandy Wanna, ${ }^{1}$ Sam Hammadi, ${ }^{1}$ Deanna Mastellone, ${ }^{1}$ KristenWilleumier ${ }^{1}$
\end{abstract}

${ }^{1}$ Department of Biomedical Sciences, UK, Germany, Netherlands, USA

${ }^{2}$ Department of Lagree Studio, USA

${ }^{3}$ Department of Pharmacology, College of Medicine and HealthSciences, UAEU

${ }^{4}$ Department Monroe Medical, UK

\begin{abstract}
The penisone of the many channels for transforming thoughts into written words. Hand writing acts as a "memorypropeller". For example, when you are at the supermarket, the simpleact of writing keeps the products you really need in your mind; or when someone at school was a shrewd knows that very often there was no need to use the sheets prepared before a classassignment to copy because they remembered everything that was written. This memory would have failed if the ticket to copy had been written or better typed on the keyboard. The latter characterizes the work of many people and yet, even today, children make their first experiences of writing with a pen or pencil. But is it really out of fashion? Today we have the opportunity to write our thoughts in other ways, "keyboard, tablet, touch screen", and even the signature, once the test par excellence of ouridentity, is faced with the competition of our " Fingerprints". Indeed, in some American schools the writing, replaced by the PC, is no longer included in the didacticplans; according to some scientists and promoters of this event, this method would facilitate children'slearning and improve creativity.
\end{abstract}

Keywords: Neuroscience, Deepskills, Recognitionmemory, Spatialmemory, Physical activity, Psychomotorskills, Lagreefitness, Mentalhealth, Rehabilitation, Cognitive exercise, Neurophysiology, Psychology, Psychophysiology

\section{Introduction}

So, what can we do? Fortunately, we come to the real "scientificresearch" based on real scientific protocols for the progress of science and not for some advertising promotion. A team of researchers coordinated by the Psychologist Sandra Sulzenbruck of the LeibnizInstitute in Dortmund hypothesizes that hand writing facilitates and stimulates "fine motorskills". The research consists of a comparison with some right-handed people who write a lot with the keyboard and other right-handed people who instead make much use of paper and pen. Both groups were asked to carry out a battery of skilltests, changing the use of the hand. Research has shown that "technology" takes longer to do the exercises but only when they use the right; it is evident that these individuals are not used to a precise coordination of movements. The mostserious effect, however, concerns above all the cognitive sphere. According to the French neuro-scientist MariekeLongcam, both children and adults find it easier to learn new signs of writing, or a new language, when they reproduce them "by hand" instead of the PC.

During the experiment some participants practiced writing the letters by hand, the others with a special keyboard. After the trial, after several weeks, the researchers verified the successes of learning; result: those who had written free hand were much better, as they were able to distinguish with greater precision the graphic gestures, learned in the previous lesson, from other similar but reversed characters. In our alphabet the reversed letters are the " $\mathrm{b}-\mathrm{d}$ - p - q"; and it is precisely on these letters that children encounter

\begin{tabular}{|l|l|}
\hline Quick Response Code: & *Corresponding author: Dario Furnari, Department of Biomedical Sciences, USA \\
Received: 23 July, 2021 & Published: 12 August, 2021 \\
Citation: Furnari D, Khan N, Delaney M, Cerna M, Hamlaoui K, et al. PEN vs. COMPUTER-How \\
to Write by Hand Improves Memory and Creativity; Neuroscience, Rehabilitation, Psychomo- \\
tor Skills. Curr Inv Cln Med Res. 2021;1(3):1-2. DOI: 10.53902/CICMR.2021.01.000512
\end{tabular}


their own difficulties especially if they are dyslexic. Also write these letters to one keyboard does not change anything from the motor point of view; since every graphic sign, for example take the "s", has a particular movement of the body, different from the act of typing on a keyboard., ${ }^{1,2}$

From the neurophysiological point of view, using fMRI (functional magnetic resonance imaging) it ispossible to visualize the graphs of brain activity. Still in the experiment, each participant who had tried to write graphic signs by hand, had memorized certain movements associated with each graph-motor sign. When they tried to remember, the tomograph showed an increase in neuronal activity in the left Broca area, in the anterior intraparietal area and in the left premotor dorsal cortex; all areas dedicated to the mental development of an action. Thus, increased motor involvement implies better "synapticplasticity" on learning, a more stable connection in memory and a better repetition of information. Now, when was the last time you used the pen? Would Manzoni or Dante have written their works anyway if they had a personal computer available? Think people think.

\section{Acknowledgments}

None.

\section{Funding}

None.

\section{Conflicts of Interest}

Authors declare that there is no conflict of interest.

\section{References}

1. Sandra Sulzenbruck, Mathias Hegele, Gerhard Rinkenauer, et al. The Death of Handwriting: SecondaryEffects of Frequent Computer Use on Basic Motor Skills. J Mot Behav. 2011.

2. Marieke Longcamp, Céline Boucard, Jean-Claude Gilhodes, et al. Learning throughHand- or Type writing influences Visual Recognition of New Graphic Shapes: Behavioral and Functional Imaging Evidence. J Cogn Neurosci. 2008;20(5):802-815. 